\title{
Contrast-enhanced spectral mammography in neoadjuvant chemotherapy monitoring: a comparison with breast magnetic resonance imaging
}

Valentina lotti ${ }^{*}$ (D), Sara Ravaioli ${ }^{1}$, Rita Vacondio ${ }^{1}$, Chiara Coriani ${ }^{1}$, Sabrina Caffarri ${ }^{2}$, Roberto Sghedoni ${ }^{3}$, Andrea Nitrosi ${ }^{3}$, Moira Ragazzi ${ }^{4}$, Elisa Gasparini ${ }^{5}$, Cristina Masini ${ }^{6}$, Giancarlo Bisagni ${ }^{6}$, Giuseppe Falco ${ }^{7}$ Guglielmo Ferrari ${ }^{7}$, Luca Braglia ${ }^{8}$, Alberto Del Prato ${ }^{1}$, Ivana Malavolti ${ }^{2}$, Vladimiro Ginocchi ${ }^{2}$ and Pierpaolo Pattacini ${ }^{1}$

\begin{abstract}
Background: Neoadjuvant-chemotherapy (NAC) is considered the standard treatment for locally advanced breast carcinomas. Accurate assessment of disease response is fundamental to increase the chances of successful breastconserving surgery and to avoid local recurrence. The purpose of this study was to compare contrast-enhanced spectral mammography (CESM) and contrast-enhanced-MRI (MRI) in the evaluation of tumor response to NAC.

Methods: This prospective study was approved by the institutional review board and written informed consent was obtained. Fifty-four consenting women with breast cancer and indication of NAC were consecutively enrolled between October 2012 and December 2014. Patients underwent both CESM and MRI before, during and after NAC. MRI was performed first, followed by CESM within 3 days. Response to therapy was evaluated for each patient, comparing the size of the residual lesion measured on CESM and MRI performed after NAC to the pathological response on surgical specimens (gold standard), independently of and blinded to the results of the other test. The agreement between measurements was evaluated using Lin's coefficient. The agreement between measurements using CESM and MRI was tested at each step of the study, before, during and after NAC. And last of all, the variation in the largest dimension of the tumor on CESM and MRI was assessed according to the parameters set in RECIST 1.1 criteria, focusing on pathological complete response (pCR).

Results: A total of 46 patients (85\%) completed the study. CESM predicted pCR better than MRI (Lin's coefficient 0.81 and 0.59 , respectively). Both methods tend to underestimate the real extent of residual tumor (mean $4.1 \mathrm{~mm}$ in CESM, $7.5 \mathrm{~mm}$ in MRI). The agreement between measurements using CESM and MRI was 0.96, 0.94 and 0.76 before, during and after NAC respectively. The distinction between responders and non-responders with CESM and MRI was identical for $45 / 46$ patients. In the assessment of CR, sensitivity and specificity were $100 \%$ and $84 \%$, respectively, for CESM, and $87 \%$ and $60 \%$ for MRI.
\end{abstract}

Conclusion: CESM and MRI lesion size measurements were highly correlated. CESM seems at least as reliable as MRI in assessing the response to NAC, and may be an alternative if MRI is contraindicated or its availability is limited.

Keywords: Breast Cancer, Neoadjuvant chemotherapy, Treatment monitoring, CESM (contrast enhanced spectral mammography), Dual-energy, Contrast media, MRI (magnetic resonance imaging)

* Correspondence: valentina.iotti@asmn.re.it

${ }^{1}$ Radiology Unit, Department of Diagnostic Imaging and Laboratory

Medicine, Arcispedale Santa Maria Nuova - IRCCS, Viale Umberto I, No. 50,

42123 Reggio Emilia, Italy

Full list of author information is available at the end of the article

(c) The Author(s). 2017 Open Access This article is distributed under the terms of the Creative Commons Attribution 4.0 International License (http://creativecommons.org/licenses/by/4.0/), which permits unrestricted use, distribution, and reproduction in any medium, provided you give appropriate credit to the original author(s) and the source, provide a link to the Creative Commons license, and indicate if changes were made. The Creative Commons Public Domain Dedication waiver (http://creativecommons.org/publicdomain/zero/1.0/) applies to the data made available in this article, unless otherwise stated. 


\section{Background}

Preoperative neoadjuvant chemotherapy (NAC) is considered the standard treatment for locally advanced breast carcinomas to observe chemosensitivity, reduce micrometastatic diseases and for down-staging of primary tumors $[1,2]$.

Response to NAC is currently assessed by combining clinical examination and conventional imaging techniques such as mammography and ultrasound (US). These methods, however, often fail to predict the final pathologic response [3]. Breast contrast-enhanced magnetic resonance imaging (MRI) has proven to be superior to mammography and US in the assessment of tumor extent and presence of additional foci (i.e. multifocality and/or multicentricity), and highly sensitive in identifying residual disease following NAC [4-6]. Contrast-enhanced spectral mammography (CESM), a promising dual-energy breast imaging technique acquired after intravenous iodine-based contrast agent, allows the evaluation of vascularized lesions in the breast, which has, up to now, been a prerogative mainly of breast MRI [7]. Compared with mammography and US, CESM improves the sensitivity for breast cancer detection without decreasing specificity [8-10]. CESM digital detector has higher spatial resolution than MRI (i.e. typically pixel sizes $100 \mu \mathrm{m}$ and $1 \mathrm{~mm}$, respectively), revealing details that are approximately 10 times better [11].

CESM is currently used for the detection of primary breast cancer, for the assessment of the extent of disease, as a problem-solving tool and as a replacement for MRI where the latter is contraindicated [12]. In terms of patient preferences and tolerance, significantly higher overall preference towards CESM has been demonstrated, due to faster procedure time, greater comfort and significantly lower rate of anxiety [13].

MRI and CESM assess treatment response by evaluating tumor size reduction and type of vascularization. In the assessment of the size of the tumor no relevant discrepancies between CESM and MRI have been observed $[11,14,15]$. However MRI involves high costs and limited availability also in industrialized countries [16]. Thus there is the rationale for substituting MRI with CESM in NAC monitoring if the performance of CESM is similar to that of MRI.

To the best of our knowledge, there are no data in the literature reporting CESM versus MRI for NAC monitoring. Therefore, the purpose of our prospective study was to compare CESM and breast MRI in the assessment of tumor response, using postoperative histologic assessment as the gold standard.

\section{Methods}

This prospective study was performed in accordance with the Declaration of Helsinki and approved by the local ethics committee. All eligible patients were willing to undergo all study procedures and provided written informed consent prior to enrolment.

\section{Patients}

From October 2012 to December 2014, 54 consenting women (mean age 54 years, range 33-72) were consecutively enrolled in this prospective study. Inclusion criteria were age 18 years or older and histologically proven breast cancer with indications for NAC. Exclusion criteria were: known BRCA-mutation (to ensure the highest radioprotection); pregnancy; contraindications to MRI or contraindications to CESM, including a history of an anaphylactoid or anaphylactic reaction to any contrast medium or impaired renal function with chronic kidney disease stage 3 or higher (creatinine clearance $<$ $60 \mathrm{ml} / \mathrm{min}$ ).

The diagnosis of breast carcinoma was based on a percutaneous tru-cut biopsy to determine the histological and molecular characteristics of the lesion detected at the initial multimodality assessment based on physical examination, digital mammography and ultrasound. The therapeutic pathway of each patient was discussed and planned by the Breast Unit Multidisciplinary Group. The NAC protocol included the administration of anthracycline for the first 3 months and then taxanes for the next 3 months; trastuzumab was added in cases of human epidermal growth factor receptor 2 (HER2) $^{+}$carcinoma. Before NAC, each patient was tattooed to ensure correct surgical positioning of the region to be excised [17].

\section{Study workflow}

The study workflow is shown in Fig. 1.

CESM and MRI were performed thrice: time ( $t$ )0, before the beginning of NAC (pre-NAC); $\mathrm{t} 3 \mathrm{~m}$, after about 3 months (during-NAC) and $t 6 \mathrm{~m}$, after the end of treatment (post-NAC), just before surgery. In premenopausal women, MRI and CESM were performed between the $5^{\text {th }}$ and $12^{\text {th }}$ day after the end of menstrual flux. MRI was performed first, and then CESM was performed within 3 days. According to the first MRI performed before the beginning of NAC, if the neoplasm involved both breasts, then CESM was performed bilaterally, otherwise it was performed only on the affected breast.

\section{CESM protocol}

CESM is based on a dual-energy system developed by GE Healthcare (Chalfont St-Giles, UK): after contrast administration, a set of low-energy and high-energy images is acquired in quick succession while the breast remains compressed, obtaining a low-dose image, comparable to a standard digital mammogram, and a post-processing recombined image, which enhances the distribution of the iodine contrast medium. The three digital mammography 


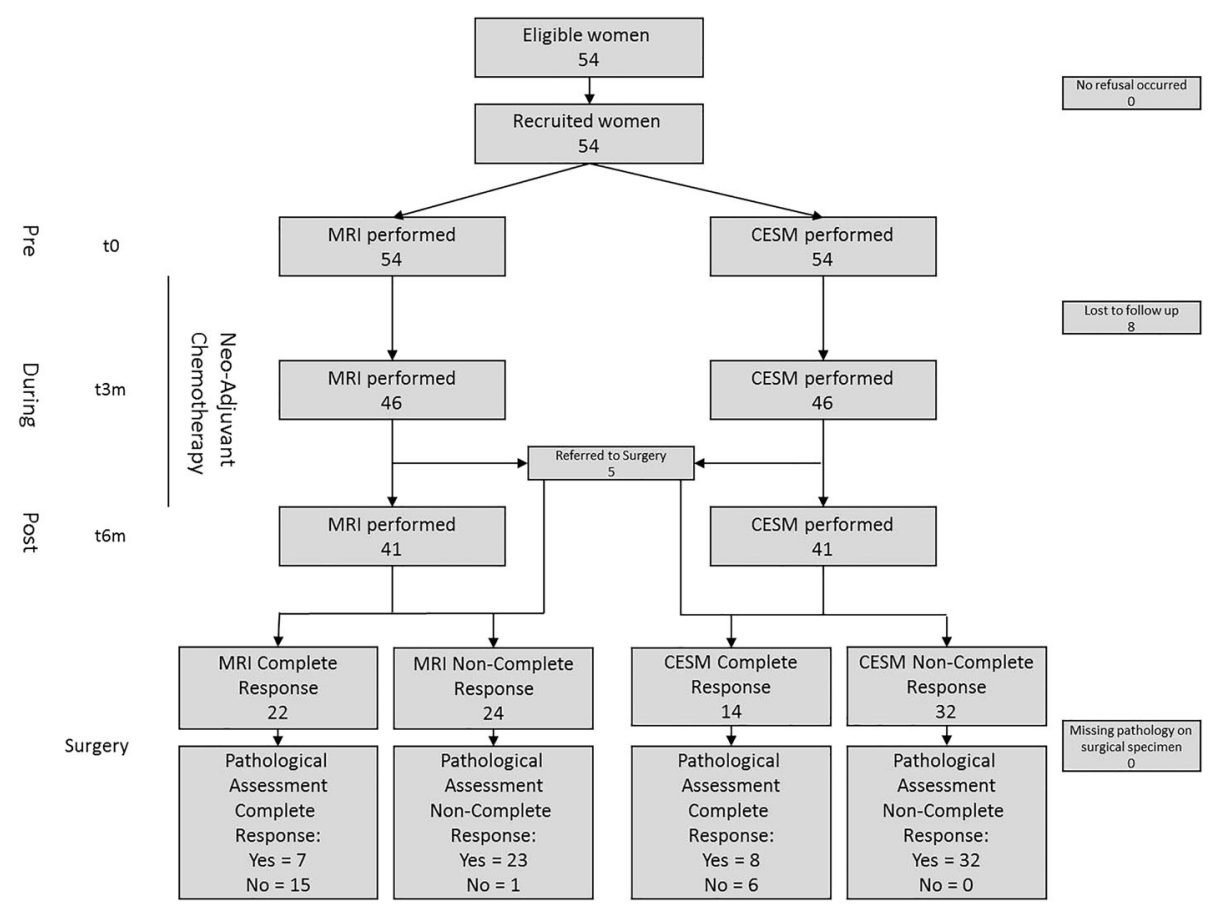

Fig. 1 Flow chart of the study. MRI magnetic resonance imaging, CESM contrast-enhanced spectral mammography

units (GE Senographe Essential) used in this study were equipped with molybdenum (Mo) and rhodium (Rh) anode tracks and filters, which can be combined to perform standard mammography. Additional copper $(\mathrm{Cu})$ filters were installed to allow the combinations of $\mathrm{Mo} / \mathrm{Cu}$ or $\mathrm{Rh} / \mathrm{Cu}$ and shape the X-ray spectra for CESM dualenergy exposures. The examinations were performed using the CESM automatic exposure option, set according to the thickness of the compressed breast and its density. The average glandular dose (AGD) delivered by the CESM examinations was evaluated.

The CESM protocol was designed according to Dromain et al. [9]. The intravenous contrast agent was administered by a nurse under the supervision of a radiologist, using a hand-held battery-powered injector (Optistat, Covidien). The contrast agent was ioversolo $350 \mathrm{mg} /$ $\mathrm{ml}$ (Optiray, Covidien) and the administered dose was $1.5 \mathrm{ml} / \mathrm{kg}$ of body weight [7-16]. In a monolateral CESM, the radiographer compressed the breast for the mediolateral oblique projection (MLO) 2 minutes after administration of contrast agent and then decompressed the breast, and after a further 2 minutes again compressed the breast for the cranio-caudal projection (CC).

\section{MRI protocol}

MRI examinations were performed with three different MRI scanners (Philips-Achieva $1.5 \mathrm{~T}$, Siemens-Avanto $1.5 \mathrm{~T}$ and GE-Signa $1.5 \mathrm{~T}$ ), all equipped with phasedarray coils (seven coils on the Philips-Achieva; eight coils on the Siemens-Avanto and GE-Signa). The same MRI protocol was applied in the prone position and with no breast compression. The sequences acquired were: T2weighted with or without fat suppression; 3D dynamic perfusion T1-weighted with or without fat suppression, with a first acquisition before contrast agent administration (mask) and seven acquisitions after the gadolinium contrast agent was administered, and temporal resolution between 50 and $70 \mathrm{~s}$; diffusion weighted sequence where $b$ values of 0 and $600 \mathrm{~mm} / \mathrm{s}^{2}$ were set. The images were then processed using commercial software (Cadstream, Merge Healthcare) [18-20]. A detailed assessment of the enhancement kinetics was evaluated by the radiologist especially during diagnosis, but its description is not an objective of the study as the comparison is based on measurements of the size of the enhanced lesions.

\section{Endpoint of the study and image analysis}

A total of seven independent radiologists, all experts in breast imaging (from 5 to 18 years' experience), took part in this study to evaluate and report CESM and MRI examinations. In the clinical workflow, the radiologist evaluated either CESM or MRI of the single patient, blinded to the MRI or CESM performed at the same step of the study, but not blinded to all previous breast examinations.

The endpoint of the study was the comparison of postNAC measurements on CESM and MRI, with the histopathological response identified in surgical specimens 
considered as the gold standard. Correlation between the measurements of the lesion using the two diagnostic tools was then evaluated for each step of the study (pre NAC, during NAC and post NAC). And last of all, the response to therapy was assessed for each patient according to the parameters set in the response evaluation criteria in solid tumors (RECIST) 1.1 criteria, considering the sum of the largest dimension of malignancies at baseline and its variation at subsequent measurements [3, 21]. Response was classified as follows: complete response (CR, disappearance of all lesions); partial response ( $\mathrm{PR}, \geq 30 \%$ dimensional reduction), stable disease $(\mathrm{SD},<30 \%$ dimensional reduction $/<20 \%$ dimensional increase) and progressive disease ( $\mathrm{PD}, \geq$ $20 \%$ dimensional increase).

In CESM measurements were obtained considering the maximum dimension on recombined images, based on contrast uptake. Since the low-energy image of CESM allows the visualization and characterization of microcalcifications, this information, integrating the extension of the enhanced area [16], contributed to defining the size of the tumor bed, especially in the pre-NAC evaluation.

In MRI, the maximum diameter of the enhancing lesion was measured on post-contrast T1-weighted or subtracted axial images at peak enhancement. For both CESM and MRI, in the evaluations during NAC and post NAC, enhancing foci spread inside the tumor bed were considered as the expression of what remained of the same pathologic area, thus defined with a single overall measure. As this evaluation is already quite common in MRI, we considered it also for CESM, according to its high sensitivity and specificity described in the literature [7-16, 22-25].

According to the literature [22, 23] and the Breast Imaging-Reporting and Data System (BI-RADS) lexicon, the lesion location and a detailed descriptive analysis of its enhancement were reported both for MRI and CESM pre NAC and post NAC: non-mass enhancement, evaluating its distribution, or mass enhancement, defining lesion shape, margins and vascularization, considering both qualitative evaluation of contrast uptake and internal enhancement patterns.

\section{Surgical specimens}

Tumor size was macroscopically measured in formalinfixed specimens. Histopathological examination was performed in paraffin-embedded specimens. Tumor size was defined as the largest dimension based on macroscopic and histopathological examination. In the case of multifocal breast cancer, the maximum dimension of the largest invasive tumor was used.

In clinical practice the persistence of residual ductal carcinoma in situ (DCIS) components is considered as pathological complete response (pCR) [26]. However, in comparing CESM and MRI measurements with histopathological response on surgical specimens, we considered the DCIS component as part of the residual neoplasm, because when visible on imaging, it impacted surgical planning.

\section{Statistical analysis}

In the absence of an a priori hypothesis, given the exploratory nature of the study, no formal sample size calculation was performed. Primary analysis included assessing agreement between CESM and MRI and histopathological assessment, and between the two diagnostic tools (pre, during and post NAC), measured with Lin's coefficient [27]. The agreement difference estimate was

Table 1 Characteristics of the patients that concluded the study

\begin{tabular}{|c|c|c|}
\hline Characteristic & Value & \\
\hline Number of included patients & 46 & \\
\hline Age, mean (range), years & $54(33-72)$ & \\
\hline Postmenopausal, $n$ (\%) & 20 & 43 \\
\hline \multicolumn{3}{|l|}{ Histological assessment, $n$ (\%) } \\
\hline Invasive ductal carcinoma & 40 & 87 \\
\hline Invasive lobular carcinoma & 4 & 9 \\
\hline Metaplastic carcinoma & 2 & 4 \\
\hline \multicolumn{3}{|l|}{ TNM stage at diagnosis, $n$ (\%) } \\
\hline T2NO & 13 & 28 \\
\hline $\mathrm{T} 2 \mathrm{~N}+$ & 17 & 37 \\
\hline T3NO & 5 & 11 \\
\hline $\mathrm{T} 3 \mathrm{~N}+$ & 9 & 20 \\
\hline $\mathrm{T} 4 \mathrm{~N}+$ & 2 & 4 \\
\hline \multicolumn{3}{|l|}{ Molecular characteristics, n (\%) } \\
\hline Luminal A & 3 & 6 \\
\hline Luminal B & 16 & 35 \\
\hline Luminal B HER2+ & 6 & 13 \\
\hline HER2+ & 9 & 20 \\
\hline Triple negative & 12 & 26 \\
\hline \multicolumn{3}{|c|}{$\begin{array}{l}\text { Histopathological response to NAC in surgical } \\
\text { specimen (RECIST 1.1), } n \text { (\%) }\end{array}$} \\
\hline Complete response (pCR) & 8 & 17 \\
\hline Partial response (pPR) & 32 & 70 \\
\hline Stable disease (pSD) & 5 & 11 \\
\hline Progressive disease (pPD) & 1 & 2 \\
\hline \multicolumn{3}{|l|}{ Surgical treatment, n (\%) } \\
\hline Lumpectomy & 28 & 61 \\
\hline Mastectomy & 18 & 39 \\
\hline
\end{tabular}

HER2 human epidermal growth factor receptor 2, NAC neoadjuvant chemotherapy, $R E C I S T$ response evaluation criteria in solid tumors, $P C R$ pathological complete response, $p P R$ pathological partial response, $p S D$ pathological stable disease, $p P D$ pathological progressive disease 


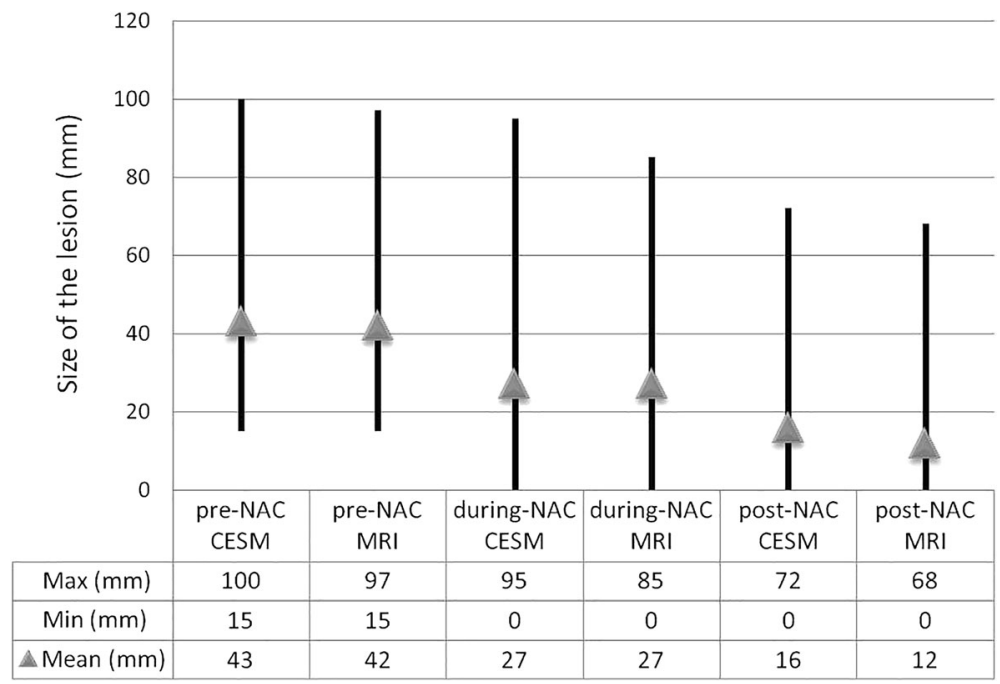

Fig. 2 Mean tumor sizes measured on contrast-enhanced spectral mammography (CESM) and magnetic resonance imaging (MRI). NAC neoadjuvant chemotherapy

accompanied by a bootstrap (bias-corrected and accelerated) confidence interval. In the first evaluation the Pearson's correlation coefficient (PCC) was also calculated to allow easier comparison with similar previous studies in the literature.

Secondary analyses included estimation of the diagnostic performance indexes of each method for complete response with 95\% confidence intervals (Clopper-Pearsonmethod [28]). Statistical analysis was carried out using R 3.2.3 (R Foundation for Statistical Computing, Vienna, Austria).

\section{Results}

Of the initial 54 patients enrolled, 46 completed the study (8/54 were excluded because of interruption in NAC before any imaging monitoring could be performed). Table 1 shows detailed patient characteristics. No multifocal or multicentric tumors were detected.

Among the 46 patients considered, as decided in the Breast Unit Multidisciplinary Group, mainly because of poor tolerance to NAC, 5 women interrupted the chemotherapy 3 months after it was started, after the second step of the imaging evaluation, and directly underwent surgery. In these patients, the second CESM and MRI have been analyzed as "post NAC" and compared to the results of assessments of the surgical specimens. Of these, according to the RECIST 1.1 criteria, two patients achieved $\mathrm{pCR}$, one $\mathrm{pPR}$, one $\mathrm{pSD}$ and one pPD. No adverse reaction was observed either to iodinated contrast medium or to gadolinium contrast agent.

In a comparison of the post-NAC measurements with the histopathological response in surgical specimens,
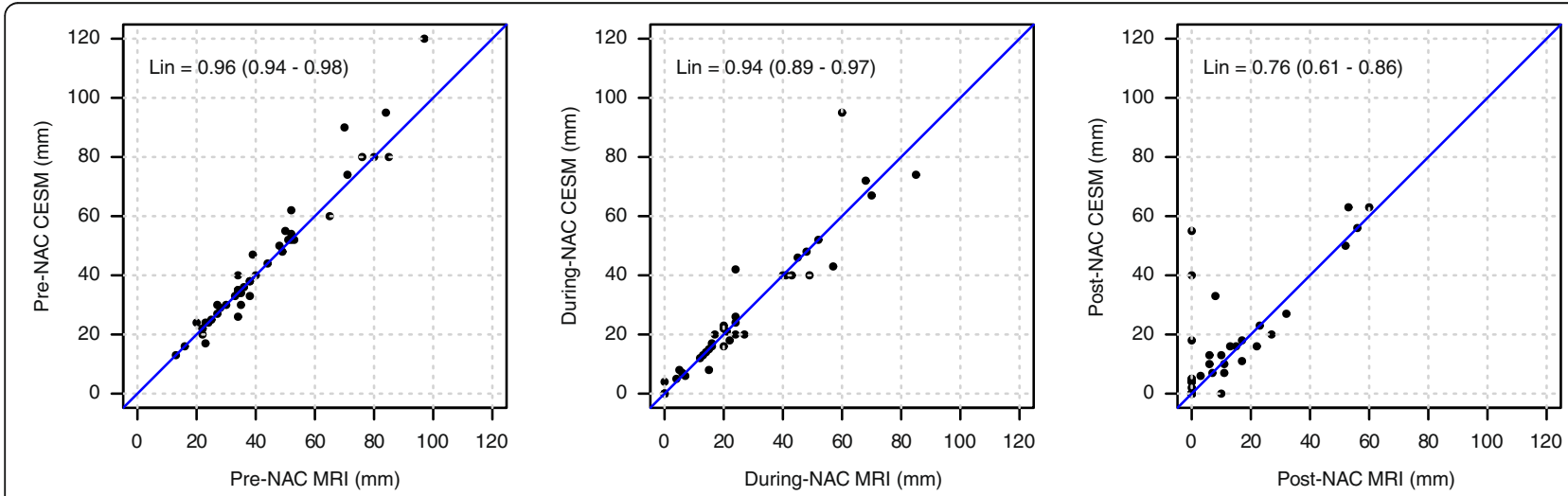

Fig. 3 Agreement between measurements on contrast-enhanced spectral mammography (CESM) and magnetic resonance imaging (MRI) at each step of the study. Agreement (Lin's coefficient) for measurement of tumor sizes using CESM and MRI (mm), respectively, before the beginning of (pre-NAC), during (during-NAC) and after the end of (post-NAC) neoadjuvant chemotherapy just before surgery. Progressive dimensional shrinkage and a difference between measurement sets using the two diagnostic tools can be observed 
Table 2 Diagnostic performance indexes in assessing complete response (CR) according to RECIST criteria 1.1, using CESM and MRI, compared to histopathological assessment of surgical specimens (gold standard)

\begin{tabular}{|c|c|c|c|c|}
\hline \multicolumn{5}{|c|}{ Assessment } \\
\hline & RECIST 1.1 & Hystopathology pCR & Hystopathology non-pCR (pPR, pSD, pPD) & \\
\hline \multirow[t]{3}{*}{ CESM } & $C R$ & 8 & 6 & PPV 57\% (Cl 29-82\%) \\
\hline & non-CR (PR, SD, PD) & 0 & 32 & NPV $100 \%$ (Cl 90-100\%) \\
\hline & & Sensitivity 100\% (Cl 63-100\%) & Specificity 84\% (Cl 69-94\%) & \\
\hline \multirow[t]{3}{*}{ MRI } & $C R$ & 7 & 15 & PPV 32\% (Cl 14-55\%) \\
\hline & non-CR (PR, SD, PD) & 1 & 23 & NPV 96\% (Cl 79-100\%) \\
\hline & & Sensitivity 87\% (Cl 47-100\%) & Specificity 60\% (Cl 43-76\%) & \\
\hline
\end{tabular}

RECIST response evaluation criteria in solid tumors, CESM contrast-enhanced spectral mammography, MRI magnetic resonance imaging, $p C R$ pathological complete response, $P P R$ pathological partial response, $P S D$ pathological stable disease, $p P D$ pathological progressive disease, $P R$ partial response, $S D$ stable disease, $P D$ progressive disease, $P P V$ positive predictive value, NPV negative predictive value

CESM had stronger agreement with histology than did MRI (Lin's coefficient 0.81 and 0.59 , respectively; CESMMRI concordance difference 0.22, CI 0.07-0.58; PCC 0.85 and 0.67 , respectively). Mean tumor sizes measured on CESM and MRI are shown in Fig. 2. Both methods tended to underestimate the real extent of the residual tumor (mean underestimation of $4.1 \mathrm{~mm}$ in CESM and of $7.5 \mathrm{~mm}$ in MRI).

Measurements on CESM image sets agreed strongly with MRI, especially before (Lin's coefficient 0.96, CI 0.94-0.98) and during (Lin's coefficient 0.94, CI 0.890.97) NAC (Fig. 3). The post-NAC agreement coefficient was 0.76 (Lin's coefficient, CI 0.61-0.86), revealing considerable divergence, which reflects the different correlation with histopathological assessment.

Post-NAC, $28 \%$ of patients had no residual pathological enhancement on CESM as compared to $46 \%$ on MRI.

According to histopathological assessment, 87\% (40/ 46) of patients responded to NAC ( $\mathrm{pCR}+\mathrm{pPR})$, with pCR achieved in 17\% (8/46). CESM defined 40 patients as responders $(C R+P R)$ while MRI defined 41 . In this single discordance, the shrinkage of tumor was $25 \%$ on CESM and $32 \%$ on MRI, close to the threshold (30\%). The main differences between CESM and MRI were observed in the distinction between CR and PR. Referring to histopathological response on surgical specimens, diagnostic performance indexes in $\mathrm{pCR}$ prediction are reported for both methods in Table 2.

On histopathological assessment 20 tumors had a DCIS component, mostly in the form of small foci in the tumor bed (Fig. 4). Of these, only three cases were classified as pCR since no concomitant residual invasive component was found: two were considered as CR both on CESM and MRI, whereas in just one case CESM revealed $5 \mathrm{~mm}$ of faint enhancement in the tumor bed, confirmed by histopathological assessment corresponding to a $3 \mathrm{~mm}$ DCIS. No surgical re-intervention was needed.

Table 3 describes the tumor characteristics at diagnosis and after NAC, according to the BI-RADS lexicon.
During and after chemotherapy, we mainly observed progressive tumor shrinkage and loss of enhancement among the responders (Fig. 5). Focusing on CESM, the shrinkage of the lesion was concentric in $60 \%$ of patients (Fig. 6), while in $40 \%$ there were multiple small foci of faint enhancement included in the tumor bed (Fig. 7).

As none of the study patients had evidence of bilateral neoplasms at the pre-NAC evaluation, the CESM examinations were always mono-lateral. The total mean AGD of a complete mono-lateral CESM, consisting of lowenergy and high-energy exposures on two mammographic views (CC and MLO), was $7.15 \mathrm{mGy}$ (range 3.88-23.34 mGy), depending on breast thickness (mean of $59 \mathrm{~mm}$, range $40-85 \mathrm{~mm}$ ) and glandularity, considered as the percentage of peak breast density measured automatically by the mammographic unit (mean of $50 \%$, range $9-95 \%$ ).

\section{Discussion}

In this prospective study we hypothesized that CESM could provide an accurate assessment of tumor response after NAC, comparable to that provided by MRI, which is currently considered the most reliable diagnostic method in treatment monitoring. In this study, the correlation between measurements using these imaging tools and histopathological assessment, the gold standard, was stronger for CESM (Lin's coefficient 0.81; PCC 0.85) than for MRI (Lin's coefficient 0.59; PCC 0.67), and both imaging tools involved slight underestimation.

In women who did not undergo NAC, Lobbes et al. [13] identified PCC $>0.9$ with both CESM and MRI compared to histopathological assessment, with a small systematic overestimation of the tumor diameter measured on MRI, but not on CESM. In a similar setting, Łuczyńska et al. [11] described overestimation of lesion size both in CESM $(1.7 \mathrm{~mm})$ and in MRI $(1.8 \mathrm{~mm})$. Fallenberg et al. [16] identified underestimation of the tumor size using MRI compared to CESM and pathological assessment, describing an increasing discrepancy 

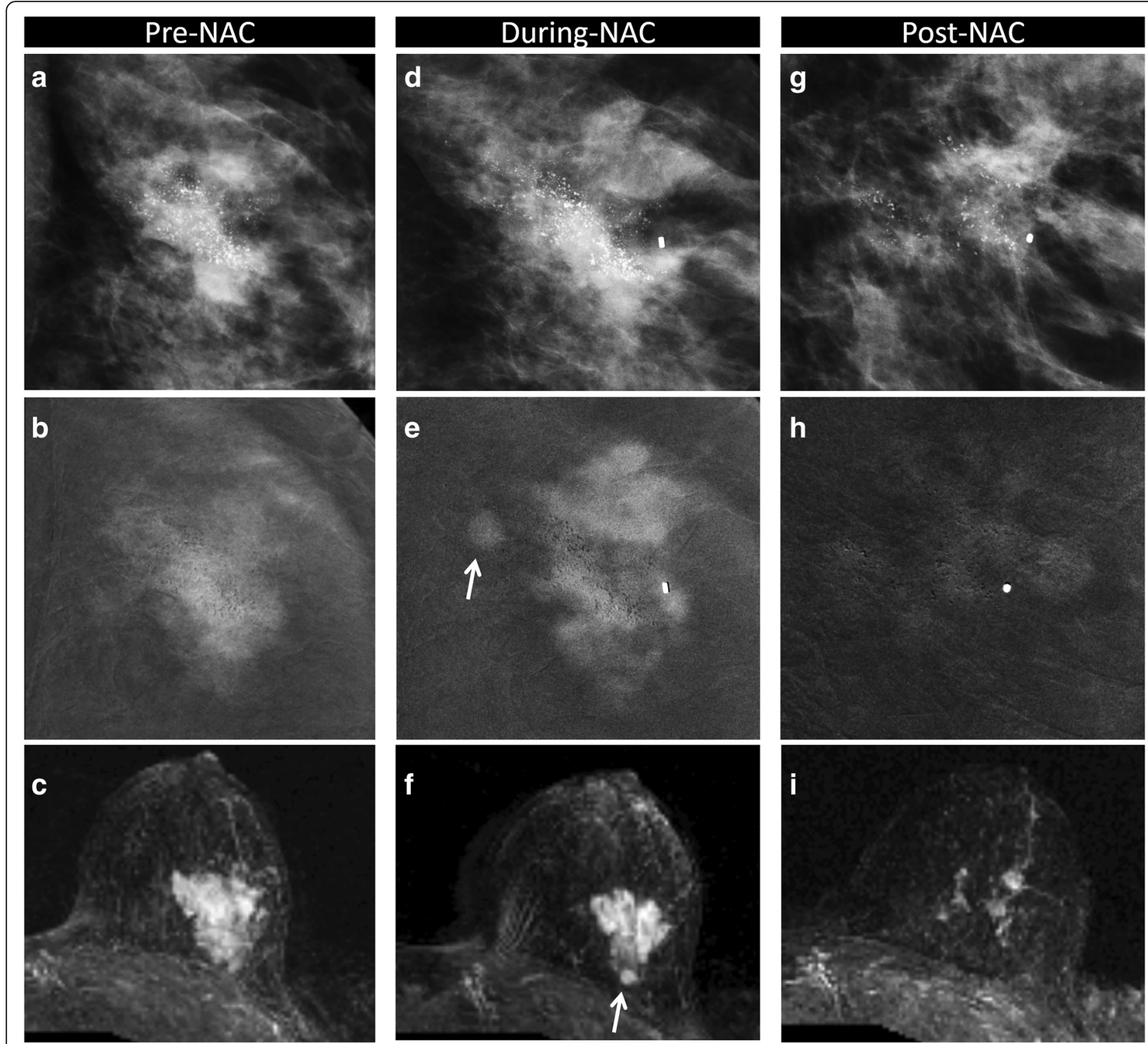

Fig. 4 Partial response in invasive ductal carcinoma (IDC) and ductal carcinoma in situ (DCIS) component with microcalcifications. Partial response to neoadjuvant chemotherapy (NAC) in a 45-year-old woman with $50 \mathrm{~mm}$ in situ and IDC (G3, luminal B) in the left breast, correctly assessed using contrast-enhance spectral mammography (CESM) (a, d, $\mathbf{g}$ magnification of craniocaudal (CC) low-dose image; $\mathbf{b}, \mathbf{e}, \mathbf{h}$ magnification of CC recombined image) and magnetic resonance imaging (MRI) (c, $\mathbf{f}, \mathbf{i}$ axial maximum intensity projection reconstruction). Pre-NAC, evaluation (a, b, $\mathbf{c})$ showed the presence of microcalcifications (a) in the context of the enhancing tumor bed on CESM (b). MRI revealed $50 \mathrm{~mm}$ of malignant enhancement (c). During-NAC ( $\mathbf{d}, \mathbf{e}, \mathbf{f})$, both on CESM ( $\mathbf{d}, \mathbf{e})$ and on MRI $(\mathbf{f})$ we identified progressive disease with the comparison of a second satellite lesion (arrow). Post NAC $(\mathbf{g}, \mathbf{h}, \mathbf{i})$, multiple foci of residual enhancement scattered inside the tumor bed were visible on CESM (g, $\mathbf{h})$ and MRI (i). Partial response was confirmed by histopathological examination of the surgical specimen

in the size of the pathological lesion in larger breast tumors measured by the imaging methods. This evaluation may also be relevant to the patients enrolled in our study, as NAC is recommended for locally advanced breast carcinomas, and at diagnosis the mean size of the lesions was $43 \mathrm{~mm}$.

Several variables influence tumor shrinkage and its evaluation during NAC, including primary tumor size, edema or necrosis. Also, the various chemotherapeutic drugs contribute differently to the variation in the primitive lesion, with significant consequences on imaging. Taxanes have been reported to have greater antiangiogenic activity compared to anthracyclines [29], and this antivascular effect, with the lack of surrounding inflammatory response, lowers the enhancement on tumor tissue, leading to underestimation of tumor size by MRI 
Table 3 Tumor characteristics at diagnosis and variations after NAC

\begin{tabular}{|c|c|c|c|c|}
\hline & Pre-NAC CESM & Pre-NAC MRI & Post-NAC CESM & Post-NAC MRI \\
\hline Mass enhancement & $89 \%(41 / 46)$ & $89 \%(41 / 46)$ & & \\
\hline \multicolumn{5}{|l|}{ Shape } \\
\hline Round & $24 \%(11 / 46)$ & $26 \%(12 / 46)$ & $15 \%(7 / 46)$ & $15 \%(7 / 46)$ \\
\hline Oval & $26 \%(12 / 46)$ & $26 \%(12 / 46)$ & $22 \%(10 / 46)$ & $11 \%(5 / 46)$ \\
\hline Irregular & $39 \%(18 / 46)$ & $37 \%(17 / 46)$ & $26 \%(12 / 46)$ & $22 \%(10 / 46)$ \\
\hline None & & & $26 \%(12 / 46)$ & $41 \%(19 / 46)$ \\
\hline \multicolumn{5}{|l|}{ Margins } \\
\hline Circumscribed & $46 \%(21 / 46)$ & $48 \%(22 / 46)$ & $35 \%(16 / 46)$ & $24 \%(11 / 46)$ \\
\hline Non-circumscribed & $43 \%(20 / 46)$ & $41 \%(19 / 46)$ & $28 \%(13 / 46)$ & $24 \%(11 / 46)$ \\
\hline None & & & $26 \%(12 / 46)$ & $41 \%(19 / 46)$ \\
\hline Non-mass enhancement & $11 \%(5 / 46)$ & $11 \%(5 / 46)$ & & \\
\hline \multicolumn{5}{|l|}{ Distribution } \\
\hline Regional & $9 \%(4 / 46)$ & $9 \%(4 / 46)$ & $7 \%(3 / 46)$ & $4 \%(2 / 46)$ \\
\hline Segmental & $2 \%(1 / 46)$ & $2 \%(1 / 46)$ & $2 \%(1 / 46)$ & $2 \%(1 / 46)$ \\
\hline None & & & $2 \%(1 / 46)$ & $4 \%(2 / 46)$ \\
\hline \multicolumn{5}{|l|}{ Enhancement pattern } \\
\hline Heterogeneous & $83 \%(38 / 46)$ & $80 \%(37 / 46)$ & $61 \%(28 / 46)$ & $43 \%(20 / 46)$ \\
\hline Clustered ring & $17 \%(8 / 46)$ & $20 \%(9 / 46)$ & $11 \%(5 / 46)$ & $11 \%(5 / 46)$ \\
\hline None & & & $28 \%(13 / 46)$ & $46 \%(21 / 46)$ \\
\hline \multicolumn{5}{|l|}{ Contrast uptake } \\
\hline Strong & $78 \%(36 / 46)$ & $83 \%(38 / 46)$ & $2 \%(1 / 46)$ & $4 \%(2 / 46)$ \\
\hline Intermediate & $22 \%(10 / 46)$ & $17 \%(8 / 46)$ & $63 \%(29 / 46)$ & $48 \%(22 / 46)$ \\
\hline Weak & & & $7 \%(3 / 46)$ & $2 \%(1 / 46)$ \\
\hline None & & & $28 \%(13 / 46)$ & $46 \%(21 / 46)$ \\
\hline
\end{tabular}

NAC neoadjuvant chemotherapy, CESM contrast-enhanced spectral mammography, MRI magnetic resonance imaging

[5]. These effects were similar in our patients as the NAC protocol was the same, including both anthracyclines and taxanes.

In this study, the cumulative impact of these variables is reflected in the progressively weaker correlation between measurements using CESM and MRI, comparing the pre-NAC (Lin's coefficient 0.96) and during-NAC (Lin's coefficient 0.94 ) evaluations to the final postNAC (Lin's coefficient 0.76). Apart from the changes in tumor micro-vessel functionality, it must be considered that the overall loss of cellularity is not always reflected by a decrease in tumor size, because the fibrous stroma remains [30]. Furthermore, when the residual cancer cells appear as small foci or scattered cells, they receive nutrients via diffusion and not from vascular perfusion [30]. Similarly, the contrast agent also moves to the ducts by diffusion, so the amount of contrast reaching the tissue is time dependent. Fallenberg et al. [16] observed that longer time delays between contrast injection and CESM exposure could result in stronger enhancement and hence better visibility compared to
MRI. In contrast to the rapid washout in MRI, enhancement on CESM in fact persists for at least $10 \mathrm{~min}$ after contrast agent infusion [15]. Further studies are encouraged to improve a protocol for CESM dedicated to NAC monitoring, especially focusing on the optimal timing in CESM acquisition after NAC to stress the viadiffusion enhancement. Other possible variables include differences in the molecular structure of gadoliniumbased and iodine-based contrast medium and the effects of breast compression. And last of all, the 10 times higher spatial resolution of CESM versus MRI [11] may help reveal tiny foci of residual enhanced lesion on the tumor bed.

According to the RECIST 1.1 criteria, the main issue considered was the distinction between $\mathrm{CR}$ and PR, with a significant divergent post-NAC assessment of no residual pathological enhancement on CESM (30\%) and on MRI (48\%). In the limited population of this study, CESM showed promising results in the definition of CR, correctly assessing each pCR, whereas one was missed on MRI. Otherwise, each patient with residual disease 


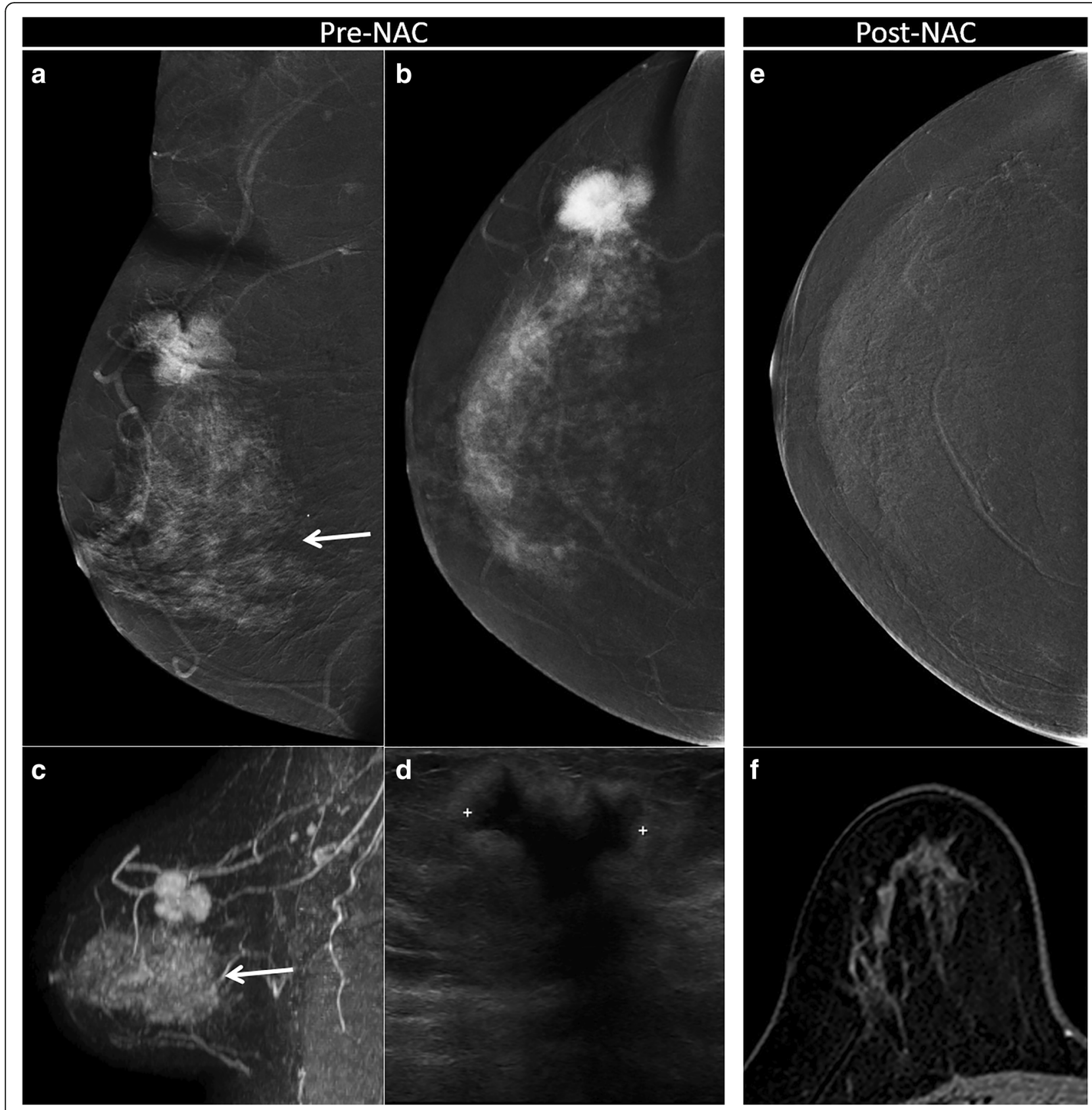

Fig. 5 Complete response correctly assessed both with contrast-enhanced spectral mammography (CESM) and magnetic resonance imaging (MRI). Complete response to neoadjuvant chemotherapy (NAC) in a 44-year-old woman with $30 \mathrm{~mm}$ in situ and invasive ductal carcinoma (G3, human epidermal growth factor receptor $\left.2(\mathrm{HER} 2)^{+}\right)$in the right breast, correctly assessed with CESM (a medio-lateral oblique recombined image; $\mathbf{b}$, e craniocaudal recombined image) and MRI (c sagittal maximum intensity projection reconstruction; $\mathbf{f}$ axial post-contrast T1-weighted at peak enhancement). Pre-NAC ( $\mathbf{a}, \mathbf{b}, \mathbf{c}, \mathbf{d})$, evaluation showed the same polygonal shape, well-defined margins and same size on CESM (a, b), on MRI (c) and on ultrasound (d). Background parenchymal enhancement (arrow) was visible both on CESM (a) and MRI (c), with no significant influence on the assessment of the size of the lesion. Post-NAC, no residual pathological enhancement was visible on CESM (e) or on MRI (f). Complete response was confirmed by histopathological examination of the surgical specimen

enhanced in CESM was confirmed on assessment of the surgical specimen.

The reliable assessment of both the extent of residual disease and pCR after NAC is crucial to decision making, surgical planning and prediction of final outcomes.
Any overestimation of lesion sizes would have less severe consequences on surgical treatment than would underestimation, especially regarding safety margins. However the underestimation of residual tumor in this study had no consequences on the surgical planning 


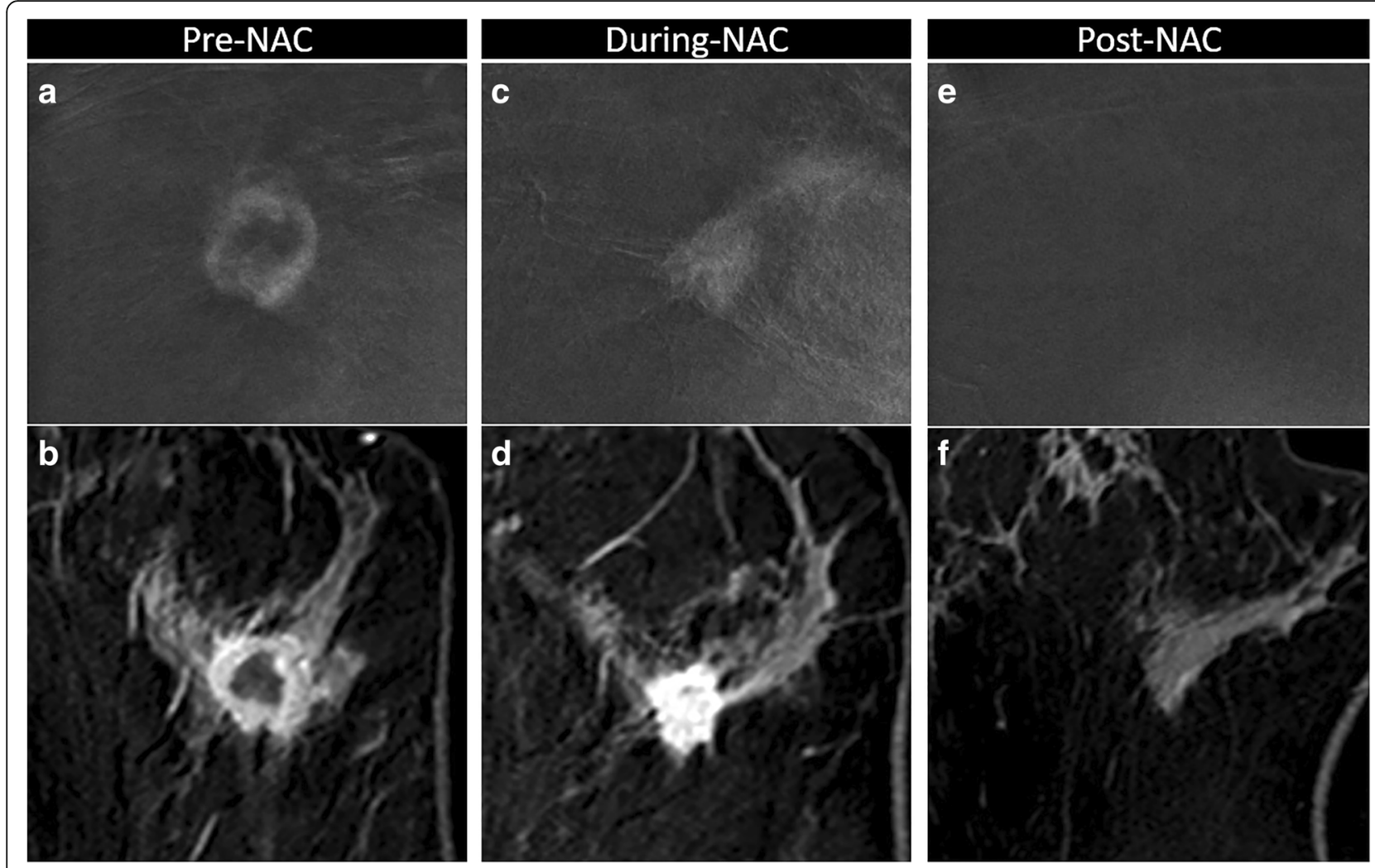

Fig. 6 Variation of enhancement during neoadjuvant chemotherapy (NAC). Complete response to NAC in a 41 -year-old woman with 27 mm invasive ductal carcinoma (G3, triple negative) in the left breast, correctly assessed with contrast-enhanced spectral mammography (CESM) ( $\mathbf{a}, \mathbf{b}, \mathbf{c}$ magnification of craniocaudal recombined image) and magnetic resonance imaging (MRI) (b, $\mathbf{d}$, $\mathbf{f}$ axial post-contrast T1-w eighted at peak enhancement). Pre-NAC, evaluation showed the same round shape, well-defined margins and rim enhancement on CESM (a) and on MRI (b). During-NAC, both on CESM (c) and on MRI (d) we noted concentric shrinkage, and the tumor was no longer rimmed, with no significant loss of enhancement intensity. Post-NAC, no residual pathological enhancement was visible on CESM (e) or on MRI (f). Complete response was confirmed by the histopathological examination of the surgical specimen

since it mainly affected the discrimination between PR and $C R$ in responding patients, in whom the tumor bed had to be completely excised anyway.

The advantages of CESM over breast MRI are the short examination time and lower costs [14]. Furthermore, CESM permits evaluation of both microcalcifications (on the low-energy image) and of enhanced structure (on the recombined image) in a single examination, overcoming this limitation of MRI and its variable sensitivity to in situ cancers $[11,31]$. Even if the persistence of residual DCIS is considered as PCR, we believe that its extent must be considered for correct surgical planning, and CESM may be a precious tool for this purpose. Further evaluations are needed. CESM might also be a good alternative if MRI is not available or for patients with contraindications to MRI (e.g. pacemakers) or claustrophobia. The tolerance and overall preference of CESM versus MRI [13] must be taken into serious consideration in women who are facing the psychological burden of chemotherapy and have to repeat the examination three times in approximately 6 months.
Disadvantages are the use of an iodine-based contrast agent instead of a gadolinium-based agent and the additional radiation dose. In this study the mean AGD of a complete mono-lateral CESM, consisting of low-energy and high-energy exposures for two mammographic views (CC and MLO), was $7.15 \mathrm{mGy}$. These doses are close to the ranges described in the literature $[9,16,32,33]$. The AGD for the low-energy image was equivalent to that of one conventional mammogram, while for the high-energy image it was approximately $20 \%$ of the dose of one conventional mammogram [9]. However, this added dose was not considered as restricting, since all patients enrolled also had indications for radiotherapy treatment, which includes a total dose of 45 Gy on the target, i.e. about 10,000 times the dose of a CESM. In this study our ethical preference was to limit CESM to the affected breast to avoid any additional dose.

This study had several limitations. First of all, the small number of patients enrolled who were not randomized, which limits the statistical significance of the results. Nevertheless, the promising findings encourage 


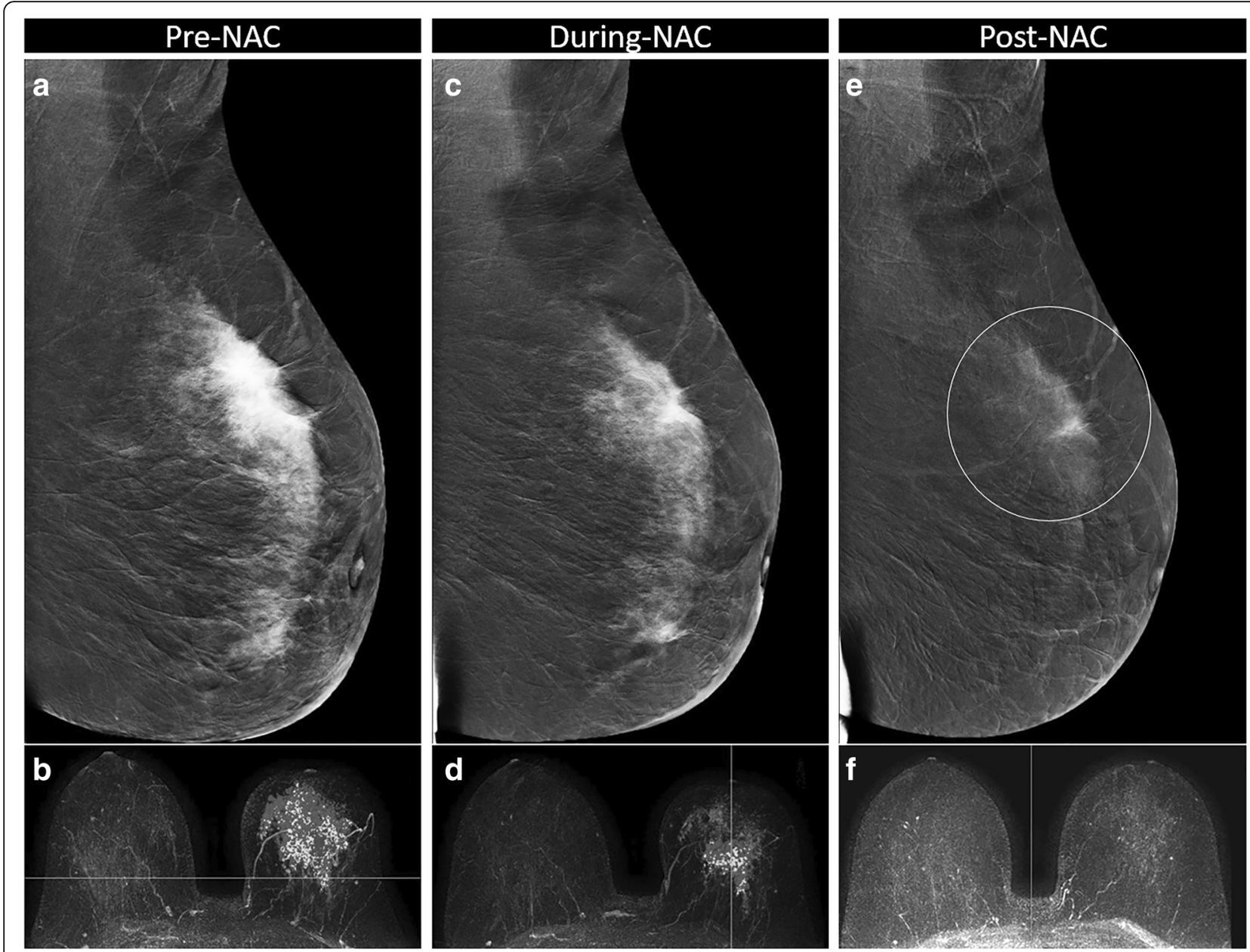

Fig. 7 Partial response correctly assessed with contrast-enhanced spectral mammography (CESM) but misinterpreted as a complete responder with MRI. Partial response to neoadjuvant chemotherapy (NAC) in a 48-year-old woman with a 97-mm invasive lobular carcinoma, luminal B, in the left breast, misinterpreted as a complete responder by MRI. Pre-NAC, evaluation showed wide non-focal enhancement on CESM (a medio-lateral oblique (MLO) recombined image) and on MRI (b axial maximum intensity projection (MIP) reconstruction). During-NAC, a concentric shrinkage and loss of enhancement intensity was visible on CESM (c MLO recombined image) and on MRI (d axial MIP reconstruction). Post-NAC, no pathological enhancement was visible on MRI (f axial MIP reconstruction), whereas in CESM (e MLO recombined image) faint enhancement in a part of the tumor bed (circle) was still visible, considered as residual tumor. The patient underwent mastectomy, and a partial response was confirmed by the histopathological examination of the surgical specimen, with $55 \mathrm{~mm}$ of residual invasive lobular carcinoma

further studies. Second, MRI was performed using different units, which may have led to heterogeneous findings despite following the same protocol. Also, the participation of seven independent radiologists might be considered a limitation because of a supposed non-homogeneous interpretation of images. However, this reflects the routine diagnostic workflow. Finally, no multicentric or multifocal breast cancers were included in the study.

\section{Conclusions}

CESM and MRI lesion size measurements were highly correlated. CESM seems at least as reliable as MRI in assessing the response to NAC, with encouraging results, and may be considered as an alternative if MRI is contraindicated or its availability is limited.

\section{Abbreviations}

AGD: Average glandular dose; BIRADS: Breast Imaging Reporting and Data System; CAD: Computer-aided detection; CC: Craniocaudal; CESM: Contrastenhanced spectral mammography; Cl: Confidence interval; CR: Complete response (RECIST 1.1 criteria); DCIS: Ductal carcinoma in situ; IDC: Invasive ductal carcinoma; HER2: Human epidermal growth factor receptor 2; MIP: Maximum intensity projection; MLO: Medio-lateral oblique:

MRI: Magnetic resonance imaging; NAC: Neoadjuvant chemotherapy; PCC: Pearson correlation coefficient; PCR: Pathological complete response (RECIST 1.1 criteria); PD: Progressive disease (RECIST 1.1 criteria); pPD: Pathological progressive disease (RECIST 1.1 criteria); pPR: Pathological partial response (RECIST 1.1 criteria); PR: Partial response (RECIST 1.1 criteria), pSD: Pathological stable disease (RECIST 1.1 criteria); RECIST: Response evaluation criteria in solid tumors; SD: Stable disease (RECIST 1.1 criteria); US: Ultrasound

\section{Acknowledgements}

We thank all the personnel for their support and devoted work during data collection, and all the women who participated in the study for their 
fundamental contribution. The authors sincerely thank Clarisse Dromain for her precious support and suggestions. The preliminary results of this study were presented orally at a scientific session and as a poster at the ECR in Vienna 2015, ECR in Vienna 2016 and at the RSNA Annual Meeting in Chicago 2016. PP, RV, SR and VI have received speakers' fees and travel grants from GE.

\section{Funding}

This work was supported by National and Regional Health Public System Funds at Arcispedale Santa Maria Nuova in Reggio Emilia and provincial Azienda USL of Reggio Emilia.

\section{Availability of data and materials}

The datasets used and/or analyzed during the present study are available from the corresponding author upon reasonable request.

\section{Authors' contributions}

SR and PP conceived and designed the study. SR, RV, CC, SC, IM and VG recruited patients and performed examinations. VI, SR, RV, CC, SC, MR, EG, CM, GB, GFa, GFe, ADP, IM and VG collected data. RS, AN and LB performed statistical analysis and interpreted the data. VI drafted the manuscript. PP critically reviewed the manuscript. All authors read and approved the final manuscript.

\section{Ethics approval and consent to participate}

All participants signed informed consent forms. The study has been approved by the provincial Ethical Committee (18/06/2012, ASMN 2012/002784-10).

\section{Consent for publication}

All authors approved the manuscript and consented to its publication. All participants signed informed consent forms for publication.

\section{Competing interests}

The authors declare that they have no competing interests.

\section{Publisher's Note}

Springer Nature remains neutral with regard to jurisdictional claims in published maps and institutional affiliations.

\section{Author details \\ ${ }^{1}$ Radiology Unit, Department of Diagnostic Imaging and Laboratory Medicine, Arcispedale Santa Maria Nuova - IRCCS, Viale Umberto I, No. 50, 42123 Reggio Emilia, Italy. ${ }^{2}$ Radiology Unit, Department of Diagnostic Imaging and Laboratory Medicine, AUSL Reggio Emilia, via Amendola 2, Reggio Emilia 42122, Italy. ${ }^{3}$ Medical Physics Unit, Department of Oncology and Advanced Technologies, Arcispedale Santa Maria Nuova - IRCCS, Viale Umberto I, No. 50, 42123 Reggio Emilia, Italy. ${ }^{4}$ Pathology Unit, Department of Oncology and Advanced Technologies, Arcispedale Santa Maria Nuova - IRCCS, Viale Umberto I, No. 50, 42123 Reggio Emilia, Italy. ${ }^{5}$ Oncology Unit, Hospital C. Magati, AUSL Reggio Emilia, Via Martiri della Libertà, No. 8, Scandiano (RE) 42019, Italy. ${ }^{6}$ Oncology Unit, Department of Oncology and Advanced Technologies, Arcispedale Santa Maria Nuova - IRCCS, Viale Umberto I, No. 50, 42123 Reggio Emilia, Italy. ${ }^{7}$ Breast Surgery Unit, Department of Surgery, Arcispedale Santa Maria Nuova - IRCCS, Viale Umberto I, No. 50, 42123 Reggio Emilia, Italy. ${ }^{8}$ Scientific Directorate, Arcispedale Santa Maria Nuova - IRCCS, Viale Umberto I, No. 50, 42123 Reggio Emilia, Italy.}

\section{Received: 1 April 2017 Accepted: 25 August 2017}

\section{Published online: 11 September 2017}

\section{References}

1. Ogston KN, Miller ID, Payne S, Hutcheon AW, Sarkar TK, Smith I, Schofield A, Heys SD. A new histological grading system to assess response of breast cancers to primary chemotherapy: prognostic significance and survival. Breast. 2003;12(5):320-7.

2. Mieog JS, van der Hage JA, van de Velde CJ. Preoperative chemotherapy for women with operable breast cancer. Cochrane Database Syst Rev. 2007;2:CD005002.

3. Semiglazov V. RECIST for response (clinical and imaging) in neoadjuvant clinical trials in operable breast cancer. J Natl Cancer Inst Monogr. 2015;2015(51):21-3. https://doi.org/10.1093/jncimonographs/lgv021.
4. Martincich L, Montemurro F, De Rosa G, Marra V, Ponzone R, Cirillo S, Gatti M, Biglia N, Sarotto I, Sismondi P, Regge D, Aglietta M. Monitoring response to primary chemotherapy in breast cancer using dynamic contrast-enhanced magnetic resonance imaging. Breast Cancer Res Treat. 2004;83(1):67-76

5. Lobbes MB, Prevos R, Smidt M, Tjan-Heijnen VC, van Goethem M, Schipper R, Beets-Tan RG, Wildberger JE. The role of magnetic resonance imaging in assessing residual disease and pathologic complete response in breast cancer patients receiving neoadjuvant chemotherapy: a systematic review. Insights Imaging. 2013;4(2):163-75. https://doi.org/10.1007/s13244-013-0219-y.

6. Sardanelli F, Boetes C, Borisch B, Decker T, Federico M, Gilbert FJ, Helbich T, Heywang-Köbrunner SH, Kaiser WA, Kerin MJ, Mansel RE, Marotti L, Martincich L, Mauriac L, Meijers-Heijboer H, Orecchia R, Panizza P, Ponti A, Purushotham AD, Regitnig P, Del Turco MR, Thibault F, Wilson R. Magnetic resonance imaging of the breast: recommendations from the EUSOMA working group. Eur J Cancer. 2010;46(8):1296-316. https://doi.org/10.1016/ j.ejca.2010.02.015.

7. Lewin JM, Isaacs PK, Vance V, Larke FJ. Dual-energy contrast enhanced digital subtraction mammography: feasibility. Radiology. 2003;229(1):261-8.

8. Dromain C, Balleyguier C, Muller S, Mathieu MC, Rochard F, Opolon P, Sigal R. Evaluation of tumor angiogenesis of breast carcinoma using contrastenhanced digital mammography. AJR Am J Roentgenol. 2006;187(5):W528-37.

9. Dromain C, Thibault F, Muller S, Rimareix F, Delaloge S, Tardivon A, Balleyguier C. Dual-energy contrast enhanced digital mammography: initial clinical results. Eur Radiol. 2011;21(3):565-74. https://doi.org/10.1007/s00330-010-1944-y.

10. Dromain C, Thibault F, Diekmann F, Fallenberg EM, Jong RA, Koomen M, Hendrick RE, Tardivon A, Toledano A. Dual-energy contrast-enhanced digital mammography: initial clinical results of a multireader, multicase study. Breast Cancer Res. 2012;14(3):R94.

11. Luczyńska E, Heinze-Paluchowska S, Hendrick E, Dyczek S, Ryś J, Herman K, Blecharz P, Jakubowicz J. Comparison between breast MRI and contrastenhanced spectral mammography. Med Sci Monit. 2015;21:1358-67. https:// doi.org/10.12659/MSM.893018.

12. Daniaux M, De Zordo T, Santner W, Amort B, Koppelstätter F, Jaschke W, Dromain C, Oberaigner W, Hubalek M, Marth C. Dual-energy contrastenhanced spectral mammography (CESM). Arch Gynecol Obstet. 2015; 292(4):739-47. https://doi.org/10.1007/s00404-015-3693-2.

13. Hobbs MM, Taylor DB, Buzynski S, Peake RE. Contrast-enhanced spectral mammography (CESM) and contrast enhanced MRI (CEMRI): patient preferences and tolerance. J Med Imaging Radiat Oncol. 2015;59(3):300-5. https://doi.org/10.1111/1754-9485.12296.

14. Lobbes MB, Lalji UC, Nelemans PJ, Houben I, Smidt ML, Heuts E, de Vries B, Wildberger JE, Beets-Tan RG. The quality of tumor size assessment by contrast-enhanced spectral mammography and the benefit of additional breast MRI. J Cancer. 2015;6(2):144-50. https://doi.org/10.7150/jca.10705.

15. Jochelson MS, Dershaw DD, Sung JS, Heerdt AS, Thornton C, Moskowitz CS, Ferrara J, Morris EA. Bilateral contrast-enhanced dual-energy digital mammography: feasibility and comparison with conventional digital mammography and MR imaging in women with known breast carcinoma. Radiology. 2013;266(3):743-51. https://doi.org/10.1148/radiol.12121084.

16. Fallenberg EM, Dromain C, Diekmann F, Engelken F, Krohn M, Singh JM, Ingold-Heppner B, Winzer KJ, Bick U, Renz DM. Contrast-enhanced spectral mammography versus MRI: initial results in the detection of breast cancer and assessment of tumour size. Eur Radiol. 2014;24(1):256-64. https://doi. org/10.1007/s00330-013-3007-7.

17. Lannin DR, Grube B, Black DS, Ponn T. Breast tattoos for planning surgery following neoadjuvant chemotherapy. Am J Surg. 2007;194(4):518-20.

18. Kuhl C. The current status of breast MR imaging. Part I. Choice of technique, image interpretation, diagnostic accuracy, and transfer to clinical practice. Radiology. 2007;244(2):356-78.

19. Kuhl CK. Current status of breast MR imaging. Part 2. Clinical applications. Radiology. 2007;244(3):672-91.

20. Levrini G, Sghedoni R, Mori C, Botti A, Vacondio R, Nitrosi A, lori M, Nicoli F. Size assessment of breast lesions by means of a computer-aided detection (CAD) system for magnetic resonance mammography. Radiol Med. 2011; 116(7):1039-49. https://doi.org/10.1007/s11547-011-0664-y.

21. Eisenhauer EA, Therasse P, Bogaerts J, Schwartz LH, Sargent D, Ford R, Dancey J, Arbuck S, Gwyther S, Mooney M, Rubinstein L, Shankar L, Dodd L, Kaplan R, Lacombe D, Verweij J. New response evaluation criteria in solid tumours: revised RECIST guideline (version 1.1). Eur J Cancer. 2009:45(2): 228-47. https://doi.org/10.1016/j.ejca.2008.10.026. 
22. Diekmann F, Freyer M, Diekmann S, Fallenberg EM, Fischer T, Bick U, Pöllinger A. Evaluation of contrast-enhanced digital mammography. Eur J Radiol. 2011;78(1):112-21. https://doi.org/10.1016/j.ejrad.2009.10.002.

23. Mohamed Kamal R, Hussien Helal M, Wessam R, Mahmoud Mansour S, Godda I, Alieldin N. Contrast-enhanced spectral mammography: Impact of the qualitative morphology descriptors on the diagnosis of breast lesions. Eur J Radiol. 2015;84(6):1049-55. https://doi.org/10.1016/j.ejrad.2015.03.005.

24. Lobbes MB, Smidt ML, Houwers J, Tjan-Heijnen VC, Wildberger JE. Contrast enhanced mammography: Techniques, current results, and potential indications. Clin Radiol. 2013;68(9):935-44. https://doi.org/10.1016/j.crad.2013.04.009.

25. Luczyńska E, Heinze-Paluchowska S, Dyczek S, Blecharz P, Rys J, Reinfuss M. Contrast-enhanced spectral mammography: comparison with conventional mammography and histopathology in 152 women. Korean J Radiol. 2014; 15(6):689-96. https://doi.org/10.3348/kjr.2014.15.6.689.

26. Cortazar $\mathrm{P}$, Zhang L, Untch M, Mehta K, Costantino JP, Wolmark N, Bonnefoi H, Cameron D, Gianni L, Valagussa P, Swain SM, Prowell T, Loibl S, Wickerham DL, Bogaerts J, Baselga J, Perou C, Blumenthal G, Blohmer J, Mamounas EP, Bergh J, Semiglazov V, Justice R, Eidtmann H, Paik S, Piccart M, Sridhara R, Fasching PA, Slaets L, Tang S, Gerber B, Geyer Jr CE, Pazdur R, Ditsch N, Rastogi P, Eiermann W, von Minckwitz G. Pathological complete response and long-term clinical benefit in breast cancer: the CTNeoBC pooled analysis. Lancet. 2014;384(9938):164-72. https://doi.org/10.1016/ S0140-6736(13)62422-8.

27. Lin LI. A concordance correlation coefficient to evaluate reproducibility. Biometrics. 1989;45(1):255-68.

28. Clopper CJ, Pearson ES. The use of confidence or fiducial limits illustrated in the case of the binomial. Biometrika. 1934;26:404-13.

29. Wu LA, Chang RF, Huang CS, Lu YS, Chen HH, Chen JY, Chang YC. Evaluation of the treatment response to neoadjuvant chemotherapy in locally advanced breast cancer using combined magnetic resonance vascular maps and apparent diffusion coefficient. J Magn Reson Imaging 2015;42(5):1407-20. https://doi.org/10.1002/jmri.24915.

30. Kim TH, Kang DK, Yim H, Jung YS, Kim KS, Kang SY. Magnetic resonance imaging patterns of tumor regression after neoadjuvant chemotherapy in breast cancer patients: correlation with pathological response grading system based on tumor cellularity. J Comput Assist Tomogr. 2012;36(2):2006. https://doi.org/10.1097/RCT.0b013e318246abf3.

31. Kim YS, Chang JM, Moon HG, Lee J, Shin SU, Moon WK. Residual mammographic microcalcifications and enhancing lesions on MRI after neoadjuvant systemic chemotherapy for locally advanced breast cancer: correlation with histopathologic residual tumor size. Ann Surg Oncol. 2016; 23(4):1135-42. https://doi.org/10.1245/s10434-015-4993-2.

32. Badr S, Laurent N, Régis C, Boulanger L, Lemaille S, Poncelet E. Dual-energy contrast enhanced digital mammography in routine clinical practice in 2013. Diagn Interv Imaging. 2014;95(3):245-58. https://doi.org/10.1016/j.diii. 2013.10.002

33. Fallenberg EM, Dromain C, Diekmann F, Renz DM, Amer H, Ingold-Heppner B, Neumann AU, Winzer KJ, Bick U, Hamm B, Engelken F. Contrast enhanced spectral mammography: does mammography provide additional clinical benefits or can some radiation exposure be avoided? Breast Cancer Res Treat. 2014;146(2):371-81. https://doi.org/10.1007/s10549-014-3023-6.

\section{Submit your next manuscript to BioMed Central and we will help you at every step:}

- We accept pre-submission inquiries

- Our selector tool helps you to find the most relevant journal

- We provide round the clock customer support

- Convenient online submission

- Thorough peer review

- Inclusion in PubMed and all major indexing services

- Maximum visibility for your research

Submit your manuscript at www.biomedcentral.com/submit
Biomed Central 